\title{
Effects of low-dose tolvaptan on electrolyte abnormality and hemodynamic parameters in a liver cirrhosis-associated portopulmonary hypertension patient: A case report
}

\author{
YOSHITO OGIHARA, NORIKAZU YAMADA, KAORU DOHI, AKIMASA MATSUDA, \\ SATOSHI OTA, KEN ISHIKURA, MASHIO NAKAMURA and MASAAKI ITO \\ Department of Cardiology and Nephrology, Mie University Graduate School of Medicine, Tsu, Mie 514-8507, Japan
}

Received November 7, 2014; Accepted October 27, 2015

DOI: $10.3892 / \mathrm{etm} .2016 .3945$

\begin{abstract}
The present study reported a case of portopulmonary hypertension (POPH) that was secondary to underlying liver cirrhosis in a 58-year-old woman, who was successfully treated with low-dose tolvaptan. The patient had suffered from refractory peripheral edema and electrolyte abnormalities, including severe hypokalemia, under the combination therapy of sildenafil, ambrisentan, furosemide and spironolactone. Subsequent to the initiation of low-dose tolvaptan at $3.75 \mathrm{mg} /$ day with concurrent de-escalation of the dose of furosemide, the daily urine volume increased, peripheral edema improved and the serum potassium level increased immediately. In addition, plasma renin activity, plasma aldosterone concentration and plasma brain natriuretic peptide level decreased within 1 week after the initiation of tolvaptan therapy. Hemodynamic assessments using a right heart catheter revealed that the pulmonary vascular resistance decreased by $\sim 20 \%$. Finally, chronic combination therapy with spironolactone and low-dose tolvaptan without loop diuretics achieved adequate fluid management. In conclusion, the findings of the present study suggest that low-dose tolvaptan may be a promising therapeutic option for liver cirrhosis-associated POPH in patients with an electrolyte abnormality due to liver cirrhosis and conventional diuretics.
\end{abstract}

\section{Introduction}

Portopulmonary hypertension (POPH) is defined as pulmonary arterial hypertension (PAH)-associated with portal hypertension. The incidence of POPH in patients with liver cirrhosis is $0.7 \%$, which increases to $16 \%$ in patients with

Correspondence to: Dr Norikazu Yamada, Department of Cardiology and Nephrology, Mie University Graduate School of Medicine, 2-174 Edobashi, Tsu, Mie 514-8507, Japan

E-mail: n-yamada@clin.medic.mie-u.ac.jp

Key words: tolvaptan, portopulmonary hypertension, electrolyte abnormality, hepatic edema, liver cirrhosis refractory ascites (1). Patients with POPH may exhibit only some of the symptoms associated with PAH, including dyspnea, hepatic ascites and edema (1). At present, therapeutic strategies for the treatment of POPH have yet to be assessed in randomized controlled trials, such that no definitive treatment is currently available.

Arterial underfilling secondary to systemic arterial vasodilatation and hypoalbuminemia-associated low oncotic pressure may lead to the activation of the renin-angiotensin-aldosterone system (RAAS) and non-osmotic release of arginine vasopressin (AVP). Activation of the RAAS and release of AVP has a major role in the pathogenesis of fluid retention and contributes to resistance to loop diuretics in patients with liver cirrhosis (2). Coexistence of liver cirrhosis with POPH may lead to elevated central venous pressure secondary to right-sided heart failure, which, in turn, adversely affects cardiorenal hemodynamics (3). Therefore, patients with liver cirrhosis and POPH often require treatment with high-dose loop diuretics that activate the RAAS and the sympathetic nervous system, which then adversely affects the cardiopulmonary circulation (2). Tolvaptan is a selective vasopressin $V_{2}$ receptor antagonist that inhibits vasopressin-mediated water reabsorption in the renal collecting ducts. It has recently been approved for the treatment of liver cirrhosis with edema (4-6). The inhibition of $\mathrm{V}_{2}$ receptor using tolvaptan is able to effectively reduce excessive fluid overload and reduce the amount of loop diuretics required, which can provide favorable neurohumoral and hemodynamic effects. The present study reports a case of POPH secondary to underlying liver cirrhosis in a 58-year-old woman, who was successfully treated with low-dose tolvaptan.

\section{Case report}

A 58-year-old woman complained of leg edema and exertional dyspnea, which was classified as World Health Organization functional class II (7). The patient had been diagnosed with hepatitis $\mathrm{C}$ virus-associated liver cirrhosis 9 years previously. The patient had also been diagnosed with POPH 3 years previously and had been treated with combination therapy of sildenafil (20 mg, three times a day) and bosentan $(62.5 \mathrm{mg}$, twice a day) for 2 years. Subsequently, bosentan was changed to ambrisentan (5 $\mathrm{mg}$, once a day) due to hepatotoxicity 1 year 
prior to admission. The patient suffered from leg edema and ascites despite combination therapy with spironolactone (100 mg/day) and furosemide ( $80 \mathrm{mg} /$ day). The patient was admitted to the Mie University Hospital (Tsu, Japan) in April 2012.

Upon hospital admission, the patient's blood pressure was $105 / 58 \mathrm{mmHg}$ with a slightly elevated pulse rate of $96 \mathrm{bpm}$ [normal range $(\mathrm{NR})=60-100 \mathrm{bpm}$ ] and $96 \%$ oxygen saturation. No signs or symptoms of hepatic encephalopathy were observed, and a physical examination showed bilateral leg edema. Cardiac auscultation showed grade $2 / 6$ pan-systolic murmur and a prominent pulmonary component of the second heart sound. A chest X-ray scan revealed pulmonary arterial dilatation and cardiomegaly, with a cardiothoracic ratio of 0.57 . Blood tests revealed pancytopenia [white blood cell count, 2,650 cells $/ \mu$ 1 ( $\mathrm{NR}=3500-9100$ cells $/ \mu \mathrm{l})$; hematocrit,33.1\% (NR=33.4-44.9\%); platelet count, $\left.3.7 \times 10^{4} / \mu \mathrm{l}\left(\mathrm{NR}=13.0-36.9 \times 10^{4} / \mu \mathrm{l}\right)\right]$, hypocoagulability [international normalized ratio of prothrombin time, $1.62(\mathrm{NR}=0.85-1.15)$ ], hyperbilirubinemia [total bilirubin, $5.5 \mathrm{mg} / \mathrm{dl}(\mathrm{NR}=0.3-1.2 \mathrm{mg} / \mathrm{dl})]$, and hypoalbuminemia $[2.7 \mathrm{~g} / \mathrm{dl}$ $(\mathrm{NR}=3.8-5.2 \mathrm{~g} / \mathrm{dl})]$; therefore, the patient was classified as having Child-Pugh class C cirrhosis (score 10) (8). The hepatic aminotransferase concentration was within the normal range and renal function was not abnormal. Severe hypokalemia and mild-moderate hyponatremia were detected, while the brain natriuretic peptide (BNP) and AVP levels in the plasma were mildly elevated. Plasma renin activity and plasma aldosterone concentration were also remarkably high. Furthermore, transthoracic echocardiography showed right ventricular and right atrial dilatation and severe tricuspid insufficiency with normal left ventricular function. A computed tomography scan showed no evidence of pulmonary embolism, interstitial lung disease or adrenal tumor, but displayed abnormal findings that suggested portal hypertension and liver cirrhosis. The respiratory function of the patient was normal. Right heart catheterization (RHC) revealed that the patient suffered from moderate PAH. In addition, the cardiac index (CI) was higher than normal, which was consistent with the diagnosis of POPH.

The patient was administered $80 \mathrm{mg} /$ day oral furosemide and $100 \mathrm{mg} /$ day spironolactone, however the mean right atrial pressure (RAP) and mean pulmonary capillary wedge pressure values ( $4 \mathrm{mmHg}$ and $8 \mathrm{mmHg}$, respectively) were not elevated. Since the patient exhibited marked hypokalemia, the dose of furosemide was decreased to $40 \mathrm{mg} /$ day following admission. In addition, low dose tolvaptan (3.75 mg/day) treatment was initiated in order to treat liver cirrhosis-associated edema and diuretic-associated adverse effects, including an electrolyte imbalance. As a result, the urine output increased to $>4,000 \mathrm{ml}$ and the leg edema was improved. Subsequently, the furosemide dose was tapered to $20 \mathrm{mg} / \mathrm{day}$. The serum potassium level was increased from 2.2 to $3.8 \mathrm{mEq} / \mathrm{dl}$, whereas the serum creatinine and blood urea nitrogen levels were not altered within 1 week of treatment and no marked changes in the patient's body weight were observed (Fig. 1 and Table I). Notably, the levels of all neurohumoral markers, including AVP, BNP, plasma renin activity (PRA), and plasma aldosterone concentration (PAC) were decreased (Table I). Subsequently, spironolactone was decreased to $50 \mathrm{mg} /$ day on day 16 following hospitalization. Repeated RHC was performed on day 17 following hospitalization (12 days after the initiation of tolvaptan treatment) and demonstrated that the mean pulmonary arterial pressure (PAP) and pulmonary vascular resistance (PVR) had decreased, although the systemic arterial pressure, mean RAP and CI remained unchanged (Table II).

The patient was discharged on day 19 after hospitalization, following a further decrease of the dose of furosemide to $10 \mathrm{mg} /$ day. The patient was successfully managed with the combination of low-dose tolvaptan and spironolactone without the need for furosemide administration 8 months after the induction of tolvaptan therapy in an outpatient setting, and serum sodium was increased (Fig. 1). The patient unfortunately died due to hepatic failure 2 years and 5 months following the induction of treatment. Written informed consent to participate in the present study was obtained from the patient's family.

\section{Discussion}

The present study reported a case of POPH that was secondary to underlying liver cirrhosis. The patient was successfully treated with low-dose tolvaptan. Tolvaptan therapy ameliorated the signs and symptoms of liver cirrhosis-associated edema, the electrolyte balance and the hemodynamic status associated with a lower dose of loop diuretics.

Arterial underfilling that is secondary to systemic arterial vasodilatation and low oncotic pressure leads to the activation of RAAS and the non-osmotic release of AVP, which plays a major role in the pathogenesis of fluid retention and contributes to resistance to loop diuretics in patients with liver cirrhosis (2). Fluid restriction in combination with orally administered aldosterone antagonists and loop diuretics has long been the primary approach used for the treatment of fluid retention and edema (2). However, these therapies may be ineffective in certain patients, and an escalating dose of furosemide induces intravenous sodium loss that contributes to decreased effective arterial blood volume, which in turn stimulates the non-osmotic release of AVP, activating the $\mathrm{V}_{2}$ receptor and inducing water reabsorption in the distal nephron (2). Free water reabsorption via AVP stimulation can cause hypervolemic hyponatremia and hypokalemia in cirrhosis $(9,10)$. Therefore, the $\mathrm{V}_{2}$ receptor is theoretically a therapeutic target. Sakaida et al $(4,5)$ and Okita et al (6) reported that add-on therapy of a low dose of tolvaptan to conventional diuretics in patients with cirrhosis was effective in improving hepatic edema with preferable tolerability. Although the aforementioned studies did not assess whether $\mathrm{V}_{2}$ receptor blockade has favorable effects on the neurohumoral system, the administration of tolvaptan was found to stabilize RAAS not only by its aquaretic potency, but also by lowering the dose of furosemide required (4-6). Indeed, PRA and PAC decreased after a short time of treatment and low-dose tolvaptan therapy was applied with concurrent de-escalation of the dose of furosemide to $20 \mathrm{mg} /$ day in present case.

Notably, the mean PAP and PVR values decreased 12 days after the initiation of tolvaptan therapy in the present case. Although no data have been previously reported regarding 
A

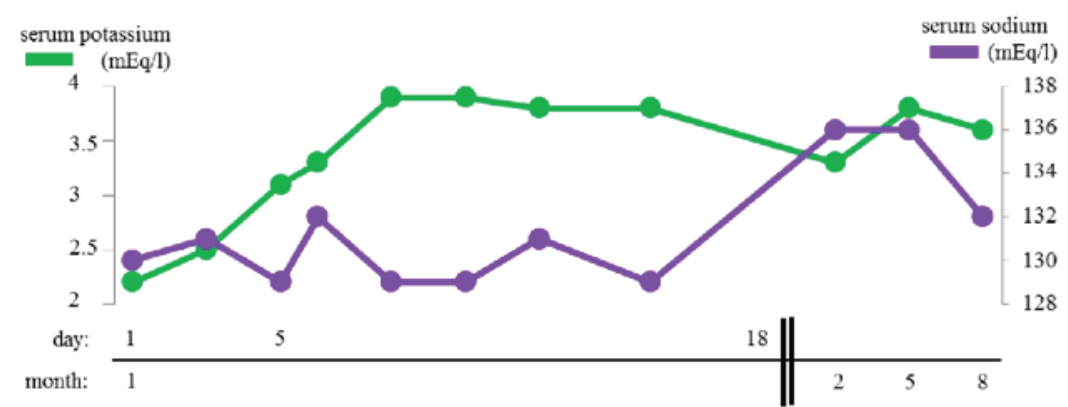

B

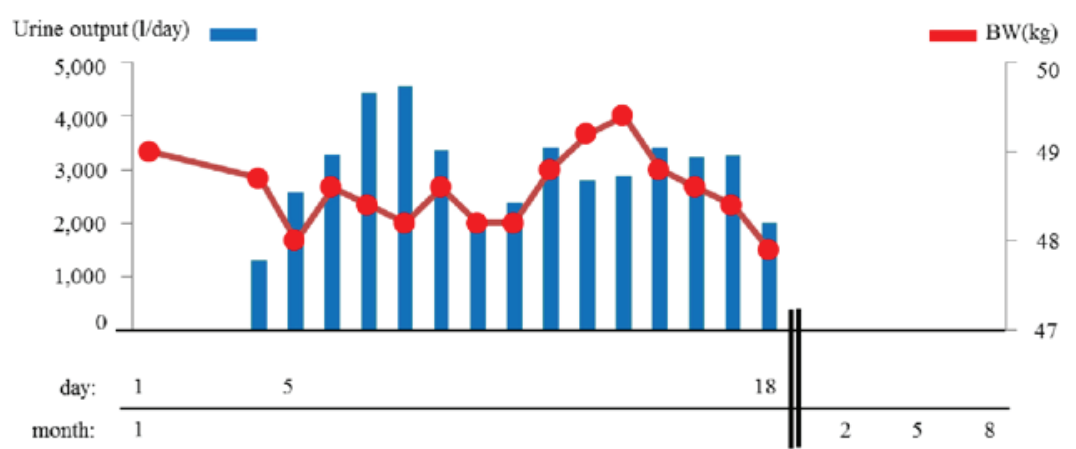

C

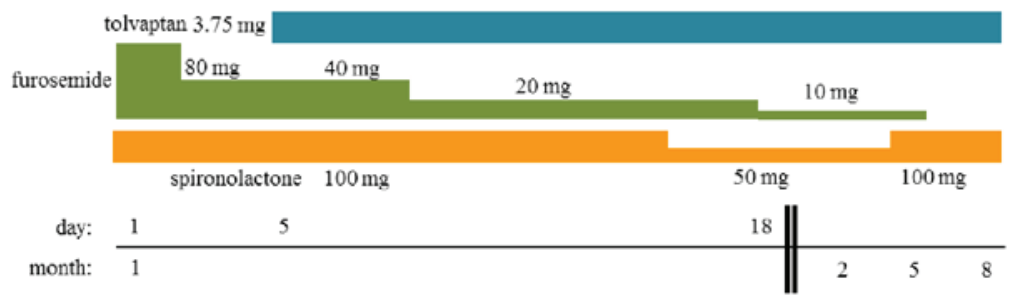

Figure 1. Summary of the clinical course of the patient. Changes in the (A) serum potassium (green line) and sodium (purple line) levels, and (B) body weight (red line) and urine output (blue bars), throughout the clinical course. (C) Various drugs that the patient received. BW, body weight.

Table I. Pertinent laboratory variables pre- and post-treatment.

\begin{tabular}{lccc}
\hline Variables & Pre-treatment & Post-treatment & Normal \\
\hline Serum sodium, mEq/l & 130 & 131 & $135-145$ \\
Serum potassium, mEq/l & 2.2 & 3.8 & $3.6-4.9$ \\
Serum urea nitrogen, $\mathrm{mg} / \mathrm{dl}$ & 15 & 12 & $8-20$ \\
Serum creatinine, $\mathrm{mg} / \mathrm{dl}$ & 0.53 & 0.46 & $0.46-0.79$ \\
AVP, $\mathrm{pg} / \mathrm{ml}$ & 4.6 & 4.2 & $<3.8$ \\
BNP, $\mathrm{pg} / \mathrm{ml}$ & 51.5 & 50 & $<18.4$ \\
Plasma renin activity, $\mathrm{ng} / \mathrm{ml} / \mathrm{h}$ & 17 & 12 & $0.3-2.9$ \\
Plasma aldosterone, $\mathrm{pg} / \mathrm{ml}$ & 463 & 391 & $29.9-159$ \\
\end{tabular}

AVP, arginine vasopressin; BNP, brain natriuretic peptide.

whether $\mathrm{V}_{2}$ receptor blockade in the distal nephron can favorably influence pulmonary circulation in patients with PAH in a clinical setting, there is increasing evidence that the RAAS plays a significant role in the development of PAH $(11,12)$. Maron et al (11) reported that plasma aldosterone levels correlate positively with the PVR and transpulmonary gradient in patients with $\mathrm{PAH}$, and Preston et al (12) reported that mineralocorticoid receptor antagonism reduced the
PVR in an experimental pulmonary hypertension model. In the present case, successful de-escalation of the furosemide dose rather than the pharmacological effect of tolvaptan may have contributed to the PVR reduction. Notably, chronic treatment combining spironolactone and low-dose tolvaptan achieved optimal fluid control without furosemide. From the neurohumoral perspective, dual blockade of the RAAS and the $\mathrm{V}_{2}$ system can play a major role in maintaining 
Table II. Clinical, right heart catheterization and blood gas variables pre- and post-treatment.

\begin{tabular}{lccc}
\hline Variables & Pre-treatment & Post-treatment & Normal \\
\hline Heart rate $(\mathrm{bpm})$ & 96 & 84 & $60-100$ \\
Blood pressure $(\mathrm{mmHg})$ & $105 / 58$ & $107 / 66$ & $<120 / 80$ \\
Mean PAP $(\mathrm{mmHg})$ & 40 & 36 & $9-19$ \\
PCWP $(\mathrm{mmHg})$ & 8 & 11 & $4-12$ \\
$\mathrm{CI}\left(\mathrm{l} / \mathrm{min} / \mathrm{m}^{2}\right)$ & 4.61 & 4.5 & $2.5-4.0$ \\
$\mathrm{SVR}(\mathrm{Wood}$ unit) & 10.7 & 11.6 & $8.75-20$ \\
PVR (Wood unit) & 4.89 & 3.91 & $1.25-3$ \\
$\mathrm{Mean}_{\mathrm{RAP}}(\mathrm{mmHg})$ & 4 & 6 & $2-7$ \\
$\mathrm{PaO}_{2}$ (mmHg) & 66.6 & 70.4 & $>80$
\end{tabular}

PAP, pulmonary arterial pressure; PCWP, pulmonary capillary wedge pressure; CO, cardiac output; CI, cardiac index; SVR, systemic vascular resistance; PVR, pulmonary vascular resistance; $\mathrm{RAP}$, right atrial pressure; $\mathrm{PaO}_{2}$, partial pressure of arterial oxygen tension.

cardiopulmonary and renal hemodynamics in patients with POPH secondary to liver cirrhosis.

In conclusion, the present study reported the use of low-dose tolvaptan in a patient with POPH and electrolyte disturbances associated with cirrhosis and conventional diuretics. A low dose of tolvaptan is considered to be pharmaceutically appropriate for such a condition, and may also provide beneficial effects on pulmonary hemodynamics without deterioration of renal and liver functions.

\section{References}

1. Hoeper MM, Krowka MJ and Strassburg CP: Portopulmonary hypertension and hepatopulmonary syndrome. Lancet 363: 1461-1468, 2004.

2. Schrier RW: Use of diuretics in heart failure and cirrhosis. Semin Nephrol 31: 503-512, 2011.

3. Golbin JM and Krowka MJ: Portopulmonary hypertension. Clin Chest Med 28: 203-218, 2007.

4. Sakaida I, Yanase M, Kobayashi Y, Yasutake T, Okada M and Okita K; ASCITES Clinical Pharmacology Group: The pharmacokinetics and pharmacodynamics of tolvaptan in patients with liver cirrhosis with insufficient response to conventional diuretics: A multicentre, double-blind, parallel-group, phase III study. J Int Med Res 40: 2381-2393, 2012.

5. Sakaida I, Kawazoe S, Kajimura K, Saito T, Okuse C, Takaguchi K, Okada M and Okita K; Ascites-Doubleblind Study Group: Tolvaptan for improvement of hepatic edema: A phase 3 , multicenter, randomized, double-blind, placebo-controlled trial. Hepatol Res 44: 73-82, 2014.

6. Okita K, Kawazoe S, Hasebe C, Kajimura K, Kaneko A, Okada M and Sakaida I; ASCITES Dose-Finding Trial Group: Dose-finding trial of tolvaptan in liver cirrhosis patients with hepatic edema: A randomized, double-blind, placebo-controlled trial. Hepatol Res 44: 83-91, 2014.
7. Galiè N, Hoeper MM, Humbert M, Torbicki A, Vachiery JL, Barbera JA, Beghetti M, Corris P, Gaine S, Gibbs JS, et al; ESC Committee for Practice Guidelines (CPG): Guidelines for the diagnosis and treatment of pulmonary hypertension: The Task Force for the Diagnosis and Treatment of Pulmonary Hypertension of the European Society of Cardiology (ESC) and the European Respiratory Society (ERS), endorsed by the International Society of Heart and Lung Transplantation (ISHLT). Eur Heart J 30: 2493-2537, 2009.

8. Pugh RN, Murray-Lyon IM, Dawson JL, Pietroni MC and Williams R: Transection of the oesophagus for bleeding oesophageal varices. Br J Surg 60: 646-649, 1973.

9. Ginés P, Berl T, Bernardi M, Bichet DG, Hamon G, Jiménez W, Liard JF, Martin PY and Schrier RW: Hyponatremia in cirrhosis: From pathogenesis to treatment. Hepatology 28: 851-864, 1998.

10. Pérez-Ayuso RM, Arroyo V, Planas R, et al: Randomized comparative study of efficacy of furosemide versus spironolactone in nonazotemic cirrhosis with ascites. Relationship between the diuretic response and the activity of the renin-aldosterone system. Gastroenterology 84: 961-968, 1983.

11. Maron BA, Opotowsky AR, Landzberg MJ, Loscalzo J, Waxman AB and Leopold JA: Plasma aldosterone levels are elevated in patients with pulmonary arterial hypertension in the absence of left ventricular heart failure: A pilot study. Eur J Heart Fail 15: 277-283, 2013.

12. Preston IR, Sagliani KD, Warburton RR, Hill NS, Fanburg BL and Jaffe IZ: Mineralocorticoid receptor antagonism attenuates experimental pulmonary hypertension. Am J Physiol Lung Cell Mol Physiol 304: L678-L688, 2013. 